\title{
Data Streams Classification by Incremental Rule Learning with Parameterized Generalization
}

\author{
Francisco Ferrer-Troyano \\ LSI, MLG, Univ. of Seville \\ Av. Reina Mercedes S/N, \\ 41012, Seville, Spain \\ ferrer@lsi.us.es
}

\author{
Jesus S. Aguilar-Ruiz \\ BIGS, Univ. Pablo de Olavide \\ Seville, Spain \\ direscinf@upo.es
}

\author{
Jose C. Riquelme \\ LSI, MLG, Univ. of Seville \\ Av. Reina Mercedes S/N, \\ 41012, Seville, Spain \\ riquelme@lsi.us.es
}

\begin{abstract}
Mining data streams is a challenging task that requires online systems based on incremental learning approaches. This paper describes a classification system based on decision rules that may store up-to-date border examples to avoid unnecessary revisions when virtual drifts are present in data. Consistent rules classify new test examples by covering and inconsistent rules classify them by distance as the nearest neighbor algorithm. In addition, the system provides an implicit forgetting heuristic so that positive and negative examples are removed from a rule when they are not near one another.
\end{abstract}

\section{INTRODUCTION}

Classification and rule learning are important, well-studied tasks in machine learning and data mining. In order to classify and model large-scale databases, important works have been recently addressed to scale up inductive classifiers and learning algorithms $[3,15]$. However, a growing number of emerging business and scientific applications, where highrate streams of detailed data are constantly generated, is frequently challenging the scalability of such methods. Examples of such data streams include networks event logs, telecommunications records, and financial and retail chain transactions. Applications of such streams include credit card fraud protection, target marketing, and intrusion detection, for which it is not possible to collect all relevant input data before applying the learning process. In these environments, KDD systems have to operate continuously - online - and process each item in real-time [4] so that memory and time limitations make multi-pass scalable algorithms unfeasible due to data are received at a higher rate than they can be repeatedly analyzed. Furthermore, realworld data streams are not generated in stationary environments, requiring incremental learning approaches to track trends and adapt to changes in the target concept.

Permission to make digital or hard copies of all or part of this work for personal or classroom use is granted without fee provided that copies are not made or distributed for profit or commercial advantage and that copies bear this notice and the full citation on the first page. To copy otherwise, to republish, to post on servers or to redistribute to lists, requires prior specific permission and/or a fee.

SAC'06, April 23-27, 2006, Dijon, France

Copyright 2006 ACM 1-59593-108-2/06/0004 ...\$5.00.
This paper describes $\mathrm{FACIL}^{1}$ (Fast and Adaptive Classifier by Incremental Learning), an incremental classifier based on decision rules that extends a previous work [1] by processing symbolic attributes. Our approach is based on filtering the examples lying near to decision boundaries so that every rule may retain both positive and negative examples. This model makes possible to ignore false alarms in order to avoid hasty modifications.

Paper Organization. The rest of the paper is organized as follows. The next section outlines a background and related work of classification, incremental learning, concept drift and data streams classification systems. In Section 3, we motivate and describe the basis of our algorithm. Section 4 describes the data sets used in our experiments and shows the results achieved. In Section 5, we discuss the conclusions we reached based on the experimental results and outline possible directions for future works.

\section{BACKGROUND}

In the problem of classification, an input data set of training examples $T=\left\{e_{1}, \ldots, e_{n}\right\}$ is given. Every training example $e_{i}=\left(\overrightarrow{x_{i}}, y_{i}\right)$ is a pair formed by a vector $\overrightarrow{x_{i}}$ and a discrete value $y_{i}$, named class label and taken of a finite set $Y$. Every vector $\overrightarrow{x_{i}}$ has the same dimensionality, each dimension is named attribute and each component $x_{i j}$ is an attribute value (numeric or symbolic). Under the assumption there is an underlying mapping function $f$ so that $y=f(\vec{x})$, the goal is to obtain a model from $T$ that approximates $f$ as $\hat{f}$ in order to classify or decide the label of non-labelled examples (tests), so that $\hat{f}$ maximizes the prediction accuracy.

Within incremental learning, a whole training set is not available a priori but examples arrives over time, normally one at a time $t$ and not time-dependent necessarily (e.g., time series). Despite online systems continuously review, update, and improve the model, not all of them are based on an incremental approach. According to the taxonomy in [12], if $T_{t}=\{(\vec{x}, y): y=f(\vec{x})\}$ for $t=\langle 1, \ldots, \infty\rangle$, then now $\hat{f}_{t}$ approximates $f$. In this context, if an algorithm discards $\hat{f}_{t-1}$ and generates $\hat{f}_{t}$ from $T_{i}$, for $i=\langle 1, \ldots t\rangle$, then it is on-line batch or temporal batch with full instance memory. If the algorithm modifies $\hat{f}_{t}$ using $\hat{f}_{t-1}$ and $T_{t}$, then it is purely incremental with no instance memory. A third approach is that of systems with partial instance memory, which select and retain a subset of past training examples to use them in future training episodes.

${ }^{1}$ Fácil is the spanish word for easy 
Along with the ordering effects, incremental learning from real-world domains faces two problems known as hidden context and concept drift, respectively [19]. The problem of hidden context is when the target concept may depend on unknown variables, which are not given as explicit attributes. In addition, hidden contexts may be expected to recur due to cyclic or regular phenomena (aka recurring contexts) [5]. The problem of concept drift is when changes in the hidden context induce changes in the target concept. In general, two kinds of concept drift depending on the rate of the changes are distinguished in the literature: sudden (abrupt) and gradual. In addition, changes in the hidden context may change the underlying data distribution, making incremental algorithms to review the current model in every learning episode. This latter problem is called virtual concept drift [19]. In [13] virtual concept drift is referred to as sampling shift, and real concept drift is referred to as concept shift. In [10] concept drift is formally defined in terms of consistency and persistence. Consistency refers to the change $\epsilon_{t}=\theta_{t}-\theta_{t-1}$ that occurs between consecutive examples of the target concept from time $t-1$ to $t$, with $\theta_{t}$ being the state of the target function in time $t$. A concept is consistent if $\epsilon_{t}$ is smaller or equal than a consistency threshold $\epsilon_{c}$. A concept is persistent if it is consistent during $p$ times, where $p \geq \frac{w}{2}$ and $w$ is the size of the window. The drift is therefore considered permanent (real) if it is both consistent and persistent. Virtual drift is consistent but it is not persistent. Noise has neither consistency nor persistence. In practice, the output model needs to be updated independently the concept drift is real or virtual.

Above problems make incremental learning be more complex than batch learning, so effective learners ought to quickly distinguish noise from actual concept drift and adapt the model to a new concept, specially in a data streams environment. Formally, a data stream is an ordered sequence of data items read in increasing order. In practice, a data stream is an unbounded sequence of items liable to both noise and concept drift, and received at a so high rate that each one can be read at most once [4]. Thus, data streams contexts compel to learning systems to give approximate answers using small and constant time per example [6].

\section{RELATED WORK}

There are two common approaches that can be applied altogether to detect changes in the target concept [8]. The former one consists in repeatedly applying the learner to a single window of training examples whose size can be dynamically adjusted whenever target function starts to drift. In [10] problems with this approach are studied and an unsupervised algorithm that uses three windows of different sizes is proposed. The second approach consists in weighting the training examples according to the time they arrive, reducing the influence of old examples. Weighting based approaches are partial instance memory methods.

Incremental rule learners based on the above approaches include STAGGER [14] (the first system designed expressly for coping with concept drift), the FLORA family [19] (with FLORA3 being the first system able to deal with recurring contexts), and the AQ-PM family [12]. A decision rule is a logic predicate of the form if antecedent then label. The antecedent is a conjunction of conditions Attribute $=$ Values where $\models$ is a operator that states a relation between a particular attribute and values of its domain.
Since fundamental rule learners take into account every training example, many of them have not still adapted to a data streams environment, especially those featuring numerical attributes. Recent works on data streams classification has been mainly addressed by two different approaches: decision trees $[2,6,7]$ and ensemble methods $[16,18]$.

Domingos \& Hulten's VFDT and CVFDT systems [6] build a decision tree based on Hoeffding bounds, which guarantee constant time and memory per example and an output model asymptotically nearly identical to that given by a batch conventional learner from enough examples. Since VFDT and CVFDT are evaluated for data streams with symbolic attributes, Jin \& Agrawal propose in [7] a numerical interval pruning approach to reduce the processing time for numerical attributes, without loss in accuracy. Gama et al.'s VFDTc system [2] extends the VFDT properties in two directions: the ability to deal with numerical attributes and the ability to apply nave Bayes classifiers in tree leaves.

Ensemble batch learning algorithms such as Boosting and Bagging have proven to be highly effective from disk-resident data sets. These techniques perform repeated resampling of the training set, making them a priori inappropriate in a data streams environment. Despite what might be expected, novel ensemble methods are increasingly gaining attention because of they have proved to offer an improvement in prediction accuracy. In general, every incremental ensemble approach uses some criteria to dynamically delete, reactivate, or create new ensemble learners in response to the base models' consistency with the current data. SEA [16] is a fast algorithm that requires approximately constant memory. It builds separate classifiers on sequential chunks of training examples, combining them into a fixed-size ensemble according to a heuristic replacement strategy. From sequential blocks as well, Wang et al. [18] propose using ensemble of classifiers weighted based on their expected classification accuracy on the test examples. In [9] Kolter \& Maloof propose DWM, an ensemble method based on the Weighted Majority algorithm [11].

As pointed out in [18], a drawback of decision trees is that even a slight drift of the target function may trigger several changes in the model and severely compromise learning efficiency. On the other hand, ensemble methods avoid expensive revisions by weighting the members, but may run the risk of building unnecessary learners when virtual drifts are present in data. Rule sets take advantage of not being hierarchically structured, so concept descriptions can be updated or removed when becoming out-of-date without hardly affecting the learning efficiency. Contrary to partitions obtained with decision tree based approaches, the regions given by decision rules do not model the whole space, so that new test examples may not satisfy - be covered by any rule.

\section{RULES WITH BORDER EXAMPLES}

Within rule learning, a rule is said consistent when does not cover any negative (different label) example. On the other hand, each training example is said a maximally specific rule. The core of our approach lies in avoiding specific rules and allowing they may be inconsistent by linking them to positive and negative examples which are very near one another (border examples). The aim is to seize border examples up to a threshold is reached. This threshold is given as an user parameter and sets the minimum pur- 
ity of a rule. The purity of a rule is the ratio between the number of positive examples that it covers and its total number of covered examples, positive and negative. When the threshold is reached, the examples associated with the rule are used to generate new positive and negative consistent rules. This approach is similar to the AQ11-PM system [12], which selects positive examples from the boundaries of its rules (hyper-rectangles) and stores them in memory. When new examples arrive, AQ11-PM combines them with those held in memory, applies the AQ11 algorithm to modify the current set of rules, and selects new positive examples from the corners, edges, or surfaces of such hyper-rectangles (extreme examples).

Our approach differs from AQ11-PM in that a rule stores two positive example per negative example covered. The stored examples are not necessary extreme and the rules are not repaired every time they become inconsistent, reducing the computational complexity. Since the number ne of negative examples that a rule can store increases as the number of covered positive examples does, every time ne increases by one unit, a new positive example is stored. Although this approach suffers the ordering effects, it does not compromise the learning efficiency and guarantees that an impure rule is always modified from as positive as negative examples.

\section{PARAMETERIZED GENERALIZATION}

Henceforth, the next notation is used to describe our proposal. Let $m$ be the number of attributes $\mathcal{A}_{j}(j \in\{1, \ldots, m\})$. Let $\mathcal{Y}=\left\{y_{1}, \ldots, y_{z}\right\}$ be the set of class labels. Let $e_{i}=$ $\left(\overrightarrow{x_{i}}, y_{i}\right)$ be the $i^{t h}$ example arriving, where $\overrightarrow{x_{i}}$ is a vector with $m$ attribute values and $y_{i}$ is a discrete value in $\mathcal{Y}$. The antecedent of a rule $r$ is given by a conjunction of $m$ conditions $\mathcal{I}_{j}$ which defines a region inside the multidimensional attribute space. $\mathcal{I}_{j}$ is a closed interval $\left[I_{j l}, I_{j u}\right]$ when $\mathcal{A}_{j}$ is a numerical attribute so that $l$ denotes lower bound and $u$ upper bound. If $\mathcal{A}_{j}$ is symbolic, then $\mathcal{I}_{j}$ is a set of values $a_{j} \in \mathcal{D}\left(\mathcal{A}_{j}\right)$ belonging to the attribute domain $\mathcal{D}\left(\mathcal{A}_{j}\right)$ and standing for a disjunction of all those values.

Definition 1 (Growth of A RULE). Let $r$ be a rule whose antecedent is formed by $m$ conditions $\mathcal{I}_{j}$. Let $e=$ $(x, y)$ be an example. The growth $\mathcal{G}(r, x)$ of the rule $r$ to cover the point $x$ is defined according to Equation 1:

$$
\begin{aligned}
& \mathcal{G}(r, x)={ }_{j=1}^{\mathrm{X}^{m}} \Delta\left(\mathcal{I}_{j}, x_{j}\right) ; \\
& \Delta\left(\mathcal{I}_{j}, x_{j}\right)=\quad \begin{array}{ll}
\delta\left(x_{j}, \mathcal{I}_{j}\right), & \text { if } \mathcal{A}_{j} \text { is numerical; } \\
\partial\left(x_{j}, \mathcal{I}_{j}\right), & \text { if } \mathcal{A}_{j} \text { is symbolic }
\end{array} \\
& \delta\left(x_{j}, \mathcal{I}_{j}\right)=\min \left(\left|I_{j l}-x_{j}\right|,\left|x_{j}-I_{j u}\right|\right) ; \\
& \partial\left(x_{j}, \mathcal{I}_{j}\right)=\begin{array}{ll}
\frac{1}{\left|\mathcal{D}\left(\mathcal{A}_{j}\right)\right|}, & \text { if } x_{j} \notin \mathcal{I}_{j} ; \\
0, & \text { if } x_{j} \in \mathcal{I}_{j} ;
\end{array}
\end{aligned}
$$

This heuristic roughly estimates the new region of the attribute space that is taken by a rule $r$ in order to describe an example $e$, biasing in favour of the rule that involves the smallest changes in the minimum number of attributes. While visiting the rules associated with $y_{i}$, the one with the minimum growth is marked as candidate. However, a rule is taken into account as a possible candidate only if the new example can be seized with a moderate growth, so that:

$$
\forall j \in\{1, \ldots, m\}: g_{j}-r_{j} \leq \kappa ; \kappa \in(0,1]
$$

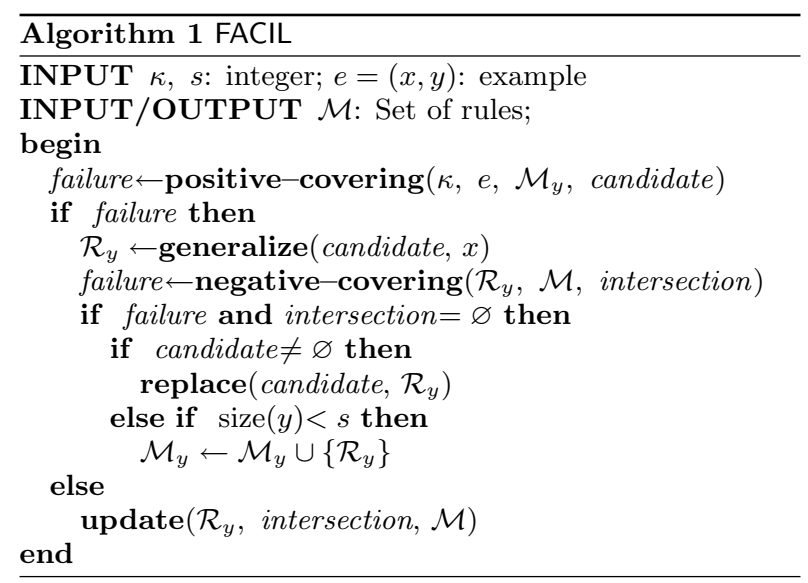

\section{LINEAR UPDATING}

Rules are stored in different sets $\mathcal{M}_{y}$ according to their associated label. Since no global training window is used but each rule handles a different set of examples (a window per rule), every time a new example $e=(\vec{x}, y)$ arrives the model is updated. In this process, three tasks are at most performed in the next order:

1. Positive covering: $x$ is covered by a rule associated with the same label $y$.

2. Negative covering: $x$ is covered by a rule associated with a different label $y^{\prime} \neq y$.

3. New description: $x$ is not covered by any rule in the model.

Positive covering. First, the rules associated with $y$ are visited and the generalization necessary to describe the new example $x$ is measured according to Equation 1.

Negative covering. If $x$ is not covered by a rule associated to $y$, then the rest of rules associated with a label $y^{\prime} \neq y$ are visited. If a different label rule $r^{\prime}$ does not cover $x$, the intersection between $r^{\prime}$ and the candidate is computed. If the intersection is not empty, the candidate is rejected. When the first different label rule $r^{\prime \prime}$ covering $x_{i}$ is found, its negative support is increased by one unit, and $x$ is added to its window. If the new purity of $r^{\prime \prime}$ is smaller than the minimum given by the user, then new consistent rules according to the examples in its window are included in the model. $r^{\prime \prime}$ is marked as unreliable so that it can not be generalized and has not taken into account to generalize other rules associated with a different label. In addition, its window is reset.

New description. After above tasks, the candidate rule is generalized if does not intersect with any other rule associated with a label $y^{\prime} \neq y_{i}$. If no rule covers the new example and there is not a candidate that can be generalized to cover it, then a maximally specific rule to describe it is generated.

Furthermore, the set of rules is simultaneously refined while the first two tasks are accomplished. Before computing a rule covers the new example, it is removed if the last extended rule associated with the same label (the last candidate) covers it. After computing a rule does not cover the new example, it is removed if satisfies one of two conditions:

- It is an unreliable rule whose support is smaller than the support of any rule generated from it. 
- The number of times the rule hindered a different label rule to be generalized is greater than its support.

\section{FORGETTING HEURISTICS}

Similarly to AQ-PM, our approach also involves a forgetting mechanism that can be either explicit or implicit. Explicit forgetting takes places when the examples are older than an user defined threshold. Implicit forgetting is performed by removing examples that are no longer relevant as they do not enforce any concept description boundary. When a negative example $x$ in a rule $r$ has not a same label example as the nearest one after the number pe of positive examples that $r$ can store is increased two times since $x$ was covered, the system removes it. Analogously, a positive example is removed if it has not a different label example as the nearest one after pe is increased by two units.

In worst case, a new example involves a new description, visiting therefore every rule in each set. The computational complexity associated with this case is $\mathcal{O}(m \cdot s \cdot \bar{e})$, with $m$ being the number of attributes and $s$ as the model size or total number of rules. $\bar{e}$ estimates the average number of examples per rule.

Finally, to classify a new test example, the systems searches the rules that cover it. If there are reliable and unreliable rules covering it, the latter ones are rejected. Consistent rules classify new test examples by covering and inconsistent rules classify them by distance as the nearest neighbour algorithm. If there is no rule covering it, the example is classified based on the label associated with the reliable rule that involves the minimum growth and does not intersect with any different label rule.

\section{EMPIRICAL EVALUATION}

Although the STAGGER concepts [14] provide a standard benchmark of tracking the drift from examples with symbolic attributes, data streams classifiers so far lacks a standard experimental method to evaluate them with numerical attributes.

In [17] an framework for incremental learning with SVMs is proposed and two incremental variants of the cross-validation experimental method are presented to evaluate them using real databases available at the UCI repository. The problem here is that both methods are designed to evaluate $\mathrm{BBL}$ algorithms (block by block learning). FACIL is based on instance by instance learning (IIL) where the algorithm does not wait for receive a block of examples - or to complete the window - to update the model, but every time a new example arrives it is processed online. Precisely, that is why standard cross validation can be applied to evaluate (IIL) learning algorithms similarly to multi-pass methods so that one-pass processing of the training examples in a sequential manner according to the order they arrive is enough.

Similarly to [17], we also evaluate our algorithm as general purpose classifier using 10-folds cross validation. Results can be found in [1]. Both experiments were conducted on a $\mathrm{PC}$ with $\mathrm{CPU} 1.7 \mathrm{GHz}$ and $512 \mathrm{MB}$ of RAM running Windows XP.

\subsection{Moving Hyperplane}

In $[6,18]$ both robustness and reliability of incremental classifiers are evaluated using synthetic data streams generated from a moving hyperplane.
A hyperplane in $\mathrm{m}$-dimensional space is denoted by equation:

$$
{ }_{i=1}^{X^{m}} a_{i} x_{i}=a_{0}
$$

First, examples are randomly generated and uniformly distributed in multidimensional space $[0,1]^{m}$. The examples satisfying $\prod_{i=1}^{m} a_{i}{\underset{p}{i}}_{i} \geq a_{0}$ are labelled as positive, and examples satisfying $\prod_{i=1}^{m} a_{i} x_{i}<a_{0}$ as negative. Weights $a_{i}$ $(1 \leq i \leq m)$ are initialized by random values in the range of $[0,1]$. The value of $a_{0}$ is chosen so that the hyperplane cuts the multi-dimensiopal space in two parts of the same volume, that is, $a_{0}=\frac{1}{2}{ }_{i=1}^{m} a_{i}$. Thus, roughly half of the examples are positive, and the other half are negative.

As in [18], concept drifts are simulated with three parameters. Parameter $\alpha$ specifies the total number of dimensions whose weights are involved in changing. Parameter $\beta \in \mathcal{R}$ specifies the magnitude of the change (every $\mathrm{N}$ examples) for weights $a_{1}, \ldots, a_{\alpha}$, and $\gamma_{i} \in\{-1,1\}$ specifies the direction of change f $f_{\beta}$ each weight. Each time the weights are updated, $a_{0}=\frac{1}{2}{ }_{i=1}^{m} a_{i}$ is recomputed so that the class distribution is not disturbed.

In addition, class noise is introduced by randomly switching the labels of $5 \%$ of the examples. As in [18], $40 \%$ dimensions' weights are changing at \pm 0.10 per 10000 examples. Figures 1-3 show the results with explicit forgetting after 100000 examples are processed. Minimum purity was set to $90 \%$. Training and test examples are generated on the fly and directly passed to the algorithm. After 900 training examples are generated, 100 test examples are used to evaluate the algorithm.

Figure 1 shows the prediction accuracy obtained by FACIL. Figure 2 shows the time in seconds spent on building the model and classifying new test examples. Figure 3 shows the final number of rules per label. Since running time depends on the number of rules, this factor is alternately limited to 50 and 100 rules per label. The goal here is evaluate the computational cost as a function of the number of attributes. Average explicit accuracy is higher than $90 \%$ and average running time is higher than 100 examples per second. However, the latter holds satisfactory tradeoffs between learning time and model complexity from low dimensionality data, so that:

- With ten attributes, learning time is greater than 3500 examples per second and accuracy exceeds $98 \%$.

- With fifty attributes, learning time is greater than 600 examples per second and accuracy exceeds $88 \%$.

\section{CONCLUSIONS AND FUTURE WORK}

FACIL is an incremental rule learner with partial instance memory based on parameterized generalization and border examples. Similarly to AQ-PM, our proposal is not based on a global window policy but examples are rejected when they do not describe a decision boundary. On the contrary, FACIL builds and refines inconsistent rules simultaneously without adversely affecting the learning efficiency since unnecessary revisions are avoided.

Our future research directions are oriented to drop irrelevant dimensions, and recover dropped attributes turned relevant later. 


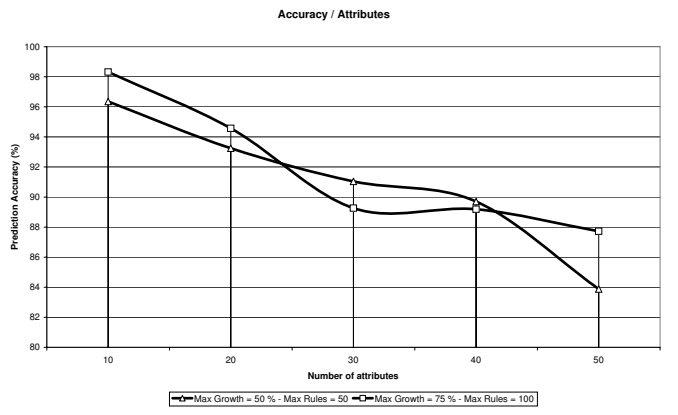

Figure 1: Prediction accuracy (\%).

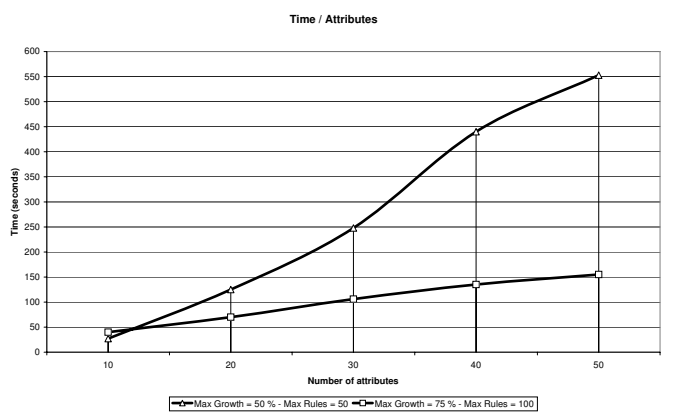

Figure 2: Time in seconds.

\section{REFERENCES}

[1] F. FerrerTroyano, J. Aguilar-Ruiz, and J. Riquelme. Incremental rule learning and border examples selection from numerical data streams. Journal of Universal Computer Science, 11(8):1426-1439, 2005.

[2] J. Gama, P. Medas, and R. Rocha. Forest trees for on-line data. In Proc. of the $19^{\text {th }}$ ACM Symposium on Applied Computing - SAC'04, pages 632-636.

[3] J. Gehrke, R. Ramakrishnan, and V. Ganti. Rainforest - a framework for fast decision tree construction of large datasets. In Proc. of the $24^{\text {th }}$ Int. Conf. on Very Large Data Bases - VLDB'98, pages 416-427, 1998.

[4] L. Golab and M. Ozsu. Issues in data stream management. SIGMOD Record, 32(2):5-14, 2003.

[5] M. Harries, C. Sammut, and K. Horn. Extracting hidden context. Machine Learning, 32(2):101-126, 1998.

[6] G. Hulten, L. Spencer, and P. Domingos. Mining time-changing data streams. In Proc. of the $7^{\text {th }} A C M$ SIGKDD Int. Conf. on Knowledge Discovery and Data Mining - KDD'01, pages 97-106.

[7] R. Jin and G. Agrawal. Efficient decision tree construction on streaming data. In Proc. of the $9^{\text {th }}$ ACM SIGKDD Int. Conf. on Knowledge Discovery and Data Mining - KDD'03.

[8] R. Klinkenberg. Learning drifting concepts: example selection vs. example weighting. Intelligent Data Analysis, Special Issue on Incremental Learning

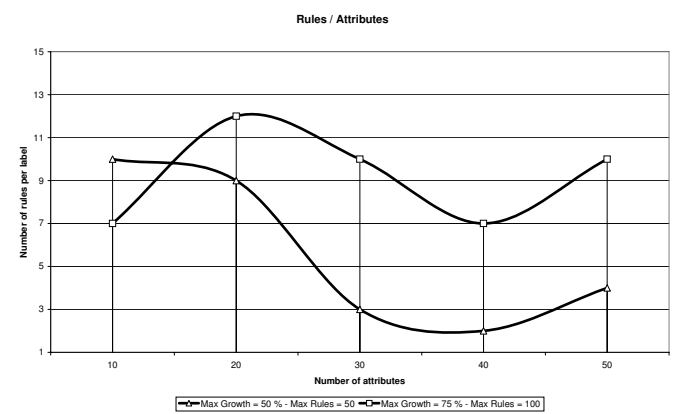

Figure 3: Number of rules.

Systems Capable of Dealing with Concept Drift, 8(3), 2004.

[9] J. Z. Kolter and M. Maloof. Dynamic weighted majority: A new ensemble method for tracking concept drift. In Proc. of the $3^{\text {th }}$ IEEE Int. Conf. on Data Mining - ICDM'03, pages 123-130, 2003.

[10] M. Lazarescu, S. Venkatesh, and H. Bui. Using multiple windows to track concept drift. Technical report, Faculty of Computer Science, Curtin University, 2003.

[11] N. Littlestone and M. Warmuth. The weighted majority algorithm. Information and Computation, 108:212-261, 1994.

[12] M. Maloof and R. Michalski. Incremental learning with partial instance memory. Artificial Intelligence, 154:95-126, 2004.

[13] M. Salganicoff. Tolerating concept and sampling shift in lazy learning using prediction error context switching. AI Review, Special Issue on Lazy Learning, 11(1-5):133-155, 1997.

[14] J. Schlimmer and R. Granger. Incremental learning from noisy data. Machine Learning, 1(3):317-354, 1986.

[15] J. Shafer, R. Agrawal, and M. Mehta. SPRINT: A scalable parallel classifier for data mining. In Proc. of the $22^{\text {th }}$ Int. Conf. on Very Large Databases $V L D B^{\prime} 96$, pages 544-555, 1996.

[16] W. Street and Y. Kim. A streaming ensemble algorithm SEA for large-scale classification. In Proc. of the $7^{\text {th }}$ ACM SIGKDD Int. Conf. on Knowledge Discovery and Data Mining - KDD'01, pages 377-382.

[17] N. Syed, H. Liu, and K. Sung. Handling concept drifts in incremental learning with support vector machines. In Proc. of the $5^{\text {th }}$ ACM SIGKDD Int. Conf. on Knowledge Discovery and Data Mining - KDD'99, pages 272-276. ACM Press, 1999.

[18] H. Wang, W. Fan, P. Yu, and J. Han. Mining concept-drifting data streams using ensemble classifiers. In Proc. of the $9^{\text {th }}$ ACM SIGKDD Int. Conf. on Knowledge Discovery and Data Mining KDD'03, pages 226-235.

[19] G. Widmer and M. Kubat. Learning in the presence of concept drift and hidden contexts. Machine Learning, 23(1):69-101, 1996. 